\title{
Abbreviated Perioperative Fasting Management for Elective Fresh Fracture Surgery: Guideline Adherence Analysis
}

\section{Zhi-jian Sun}

Beijing Jishuitan Hospital

\section{Xu Sun}

Beijing Jishuitan Hospital

Yan Huo

Beijing Jishuitan Hospital

Meng Mi

Beijing Jishuitan Hospital

Gui-ling Peng

Beijing Jishuitan Hospital

Chun-ling Zhang

Beijing Jishuitan Hospital

Yao Jiang

Beijing Jishuitan Hospital

Yan Zhou

Beijing Jishuitan Hospital

Xia Zhao

Beijing Jishuitan Hospital

Ting Li ( liting2000@sina.com )

Beijing Jishuitan Hospital

\section{Xin-bao Wu}

Beijing Jishuitan Hospital

\section{Research Article}

Keywords: perioperative fasting, compliance, fresh fractures, enhanced recovery after surgery

Posted Date: January 3rd, 2022

DOI: https://doi.org/10.21203/rs.3.rs-1114717/v1 
License: (c) (i) This work is licensed under a Creative Commons Attribution 4.0 International License. Read Full License 


\section{Abstract}

\section{Background}

Long-term fasting for elective surgery has been proven unnecessary based on established guidelines. Instead, preoperative carbohydrate loading 2 hours before surgery and recommencing oral nutrition intake as soon as possible after surgery is recommended. This study was performed to analyze the compliance with and effect of abbreviated perioperative fasting management in patients undergoing surgical repair of fresh fractures based on current guidelines.

\section{Methods}

Patients with fresh fractures were consecutively enrolled from May 2019 to July 2019 at our hospital. A carbohydrate-enriched beverage was recommended up to 2 hours before surgery for all surgical patients except those with contraindications. Postoperatively, oral clear liquids were allowed once the patients had regained full consciousness, and solid food was allowed 1 to 2 hours later according to the patients' willingness. The perioperative fasting time was recorded and the patients' subjective comfort with respect to thirst and hunger was assessed using an interview-assisted questionnaire.

Results

In total, 306 patients were enrolled in this study. The compliance rate of preoperative carbohydrate loading was $71.6 \%$, and $93.5 \%$ of patients began ingestion of oral liquids within 2 hours after surgery. The median (interquartile range) preoperative fasting time for liquids and solids was 8 (5.2-12.9) and 19 (15.7-22) hours, respectively. The median postoperative fasting time for liquids and solids was 1 (0.5$1.9)$ and $2.8(2.2-3.5)$ hours, respectively. A total of $70.3 \%$ and $74.2 \%$ of patients reported no thirst or hunger during the perioperative period. Logistic regression analysis showed that the preoperative fasting time for liquids was an independent risk factor for perioperative hunger. No adverse events such as aspiration pneumonia or gastroesophageal reflux were observed.

\section{Conclusions}

In this study of a real clinical practice setting, abbreviated perioperative fasting management was carried out with high compliance in patients with fresh fractures. The preoperative fasting time should be further shortened to further improve patients' subjective comfort.

\section{Background}

Perioperative fasting is required for elective surgery to prevent aspiration pneumonia, nausea, vomiting, and dysphagia ${ }^{[1]}$. However, long-term fasting not only worsens patients' discomfort by increasing thirst, hunger, anxiety, and fatigue but also deteriorates insulin resistance ${ }^{[2-4]}$. Many guidelines have recommended access to clear liquids and solids for up to 2 and 6 hours, respectively, before elective surgery ${ }^{[5-8]}$. Furthermore, a preoperative carbohydrate-enriched beverage has been highly recommended 
to reduce patients' discomfort, the impact of postoperative insulin resistance, and the length of hospital stay ${ }^{[8-11]}$.

The consensus on postoperative fasting is that interruption of oral nutrition intake after surgery is usually unnecessary. Oral liquids and food should be recommenced as soon as possible according to the patients' tolerance ${ }^{[7,8,12]}$. However, because of significant variations among types of surgeries performed, the exact time at which oral intake should be recommenced postoperatively is absent in common guidelines. In 2019, the Chinese guideline of perioperative fasting management in orthopedic surgery recommended resuming oral clear liquids once patients have regained their awareness (as assessed by the Steward score) and then transiting to a normal diet in the following 1 to 2 hours, considering that the gastrointestinal tract is seldom involved in orthopedic surgeries ${ }^{[13]}$.

Nevertheless, compliance with abbreviated perioperative fasting is usually poor because of uncertainty regarding the operation starting time, surgeons' and anesthetists' viewpoints, patients' willingness, and similar influencing factors ${ }^{[14-17]}$. Moreover, most studies have only reported the preoperative fasting status; postoperative oral nutrition recommencement has rarely been studied ${ }^{[18-20]}$. Thus, we conducted this study to analyze the compliance with and effect of abbreviated perioperative fasting management in patients undergoing surgical treatment of fresh fractures.

\section{Methods}

\section{Participants}

Patients with fresh fractures were consecutively enrolled from May 2019 to July 2019 at our hospital according to the following criteria.

The inclusion criteria were an age of $\geq 14$ years with no sex limitation; an upper or lower extremity, pelvic, or acetabular fracture with a single part; a fresh fracture ( $<3$ weeks); and voluntary participation in this study with provision of written informed consent.

The exclusion criteria were multiple trauma; open fractures; mental disorders, alcohol dependence, or a history of drug abuse; pregnancy or lactation; allergic constitution or allergy to a variety of drugs; and lack of suitability for this study as judged by researchers.

This study was approved by the ethics committee of Beijing Jishuitan Hospital (201807-12). And the research was performed in accordance with relevant guidelines. Informed consent was obtained from all participants.

Perioperative fasting protocol

Abbreviated perioperative fasting management was conducted according to the concept of enhanced recovery after surgery (ERAS), which was introduced at our trauma center in 2016. Clear liquids were 
recommended up to 2 hours before surgery for all surgical patients except those with delayed gastric emptying associated with conditions such as emergency surgery, gastrointestinal obstruction disease, upper gastrointestinal cancer, morbid obesity, pregnancy, gastroesophageal reflux, diabetes mellitus, and inability to engage in oral feeding. A carbohydrate-enriched beverage containing $12.5 \%$ maltodextrin (made by the nutritional department of our hospital) was highly recommended ${ }^{[5,8]}$. Patients were allowed to drink $800 \mathrm{~mL}$ of this beverage the night before surgery and no more than $400 \mathrm{~mL}$ the day of surgery up to 2 hours prior to surgery. Patient receipt of the carbohydrate-enriched beverage preoperatively on the day of surgery was defined as compliance with preoperative carbohydrate loading. Postoperatively, the anesthetists assessed the patients' consciousness using the Steward score, and oral clear liquids were allowed when the Steward score reached $\geq 5$. Solid food was then allowed 1 to 2 hours later according to the patients' willingness $^{[4]}$.

\section{Data collection}

All data were collected prospectively. The collected data included patients' basic information [age, sex, body mass index (BMI), fracture type (upper extremity fracture, lower extremity fracture, or pelvic/acetabular fracture), and comorbidities (hypertension, diabetes mellites, or other conditions)] and surgical information [anesthesia type (general anesthesia and spinal/regional blocking anesthesia), surgery type (open reduction with internal fixation, closed reduction with internal fixation, and others), and surgical time]. The perioperative fasting times, including the preoperative liquid fasting time, preoperative solid fasting time, postoperative liquid fasting time, and postoperative solid fasting time, were also collected. The total fasting time for liquids and solids was then calculated. Patients' subjective comfort with respect to thirst and hunger (ranked as none, some, or severe) was assessed using an interviewassisted questionnaire.

Statistical analysis

Statistical analysis was performed using IBM SPSS Statistics for Windows, Version 25.0 (IBM Corp., Armonk, NY, USA). Quantitative data are presented as mean \pm standard deviation or median (interquartile range), and categorical data are presented as number (percentage). For group comparisons of quantitative data, Student's $t$ test and the Mann-Whitney $U$ test were used for normally and non-normally distributed data, respectively. For group comparisons of categorical data, the chi-square test was used. Taking patients' subjective comfort with respect to thirst and hunger as a dependent variable, logistic regression tests were performed to identify relevant risk factors. A $P$ value of $<0.05$ was considered statistically significant.

\section{Results}

Compliance analysis

In total, 306 patients were enrolled in this study. The patients comprised 183 (59.8\%) men and 123 $(40.2 \%)$ women with a mean age of $43.7 \pm 16.0$ years. A total of 219 patients underwent preoperative 
carbohydrate loading the day before and the day of surgery (compliance rate of $71.6 \%)$. Ten (3.3\%) patients did not drink the carbohydrate-enriched beverage because of contraindications. Seventy-seven (25.2\%) patients had true non-compliance with abbreviated preoperative fasting. For postoperative fasting, $286(93.5 \%)$ patients regained oral fluids within 2 hours after surgery. No adverse events, such as aspiration pneumonia or gastroesophageal reflux, were observed.

Fasting time

The median preoperative fasting time for liquids and solids was 8 (5.2-12.9) and 19 (15.7-22) hours, respectively. The median postoperative fasting time for liquids and solids was 1 (0.5-1.9) and 2.8 (2.23.5) hours, respectively. The total perioperative fasting time for liquids and solids was $11(8-15.7)$ and $23.7(20.8-26.5)$ hours, respectively.

When the patients were divided according to preoperative carbohydrate loading compliance, the preoperative and total fasting times for liquids were significantly shorter in the compliance group $(P<$ $0.05)$, whereas the postoperative fasting time for liquids was longer in the compliance group $(P>0.05)$ (Table 1). The fasting time for solids was not significantly different $(P>0.05)$. The patients' age, sex, fracture type, $\mathrm{BMI}$, comorbidities, anesthesia type, surgery type, and surgical time also showed no significant differences between the two groups $(P>0.05)$.

Table 1. Comparison of perioperative fasting time between preoperative carbohydrate loading compliance and non-compliance groups

\begin{tabular}{lcccc}
\hline Fasting time & $\begin{array}{c}\text { Compliance group } \\
(\mathrm{N}=219)\end{array}$ & $\begin{array}{c}\text { Non-compliance } \\
\text { group (N=87) }\end{array}$ & $\mathrm{Z}$ & $P$ \\
\hline $\begin{array}{l}\text { Preoperative for liquids (hours, median, } \\
\text { interquartile range) }\end{array}$ & $6.8(4.3,8.5)$ & $15.5(12,18.5)$ & 11.862 & $\square 0.0001$ \\
\hline $\begin{array}{l}\text { Preoperative for solids (hours, median, } \\
\text { interquartile range) }\end{array}$ & $18.8(15.6,21.8)$ & $19(16,22.5)$ & 0.757 & 0.449 \\
$\begin{array}{l}\text { Postoperative for liquids (hours, median, } \\
\text { interquartile range) }\end{array}$ & $1(0.6,2)$ & $0.7(0.5,1.5)$ & 2.136 & 0.033 \\
$\begin{array}{l}\text { Postoperative for solids (hours, median, } \\
\text { interquartile range) }\end{array}$ & $2.9(2.3,3.5)$ & $2.6(2,3.5)$ & 1.628 & 0.104 \\
$\begin{array}{l}\text { Total for liquids (hours, median, } \\
\text { interquartile range) }\end{array}$ & $9.5(7.1,12)$ & $18(14.5,21)$ & 11.049 & $\square 0.0001$ \\
$\begin{array}{l}\text { Total for solids (hours, median, } \\
\text { interquartile range) }\end{array}$ & $23.7(20.9,26.7)$ & $23.5(20.5,26)$ & 0.274 & 0.784 \\
\hline
\end{tabular}

Subjective comfort analysis

In total, 215 (70.3\%) and 227 (74.2\%) patients had no thirst and hunger, respectively, during the perioperative period; $87(28.4 \%)$ and $72(23.5 \%)$ patients felt some thirst and hunger, respectively; and 4 $(1.3 \%)$ and $7(2.3 \%)$ patients had severe thirst and hunger, respectively. Logistic regression analysis showed that the perioperative fasting time for liquids was an independent risk factor for perioperative hunger $(P<0.05)$.

\section{Discussion}


Many guidelines have already recommended abbreviated perioperative fasting for patients undergoing elective surgery. However, changes in real clinical practice are usually slow. With the development of the ERAS protocol, shortening of the perioperative fasting time and preoperative carbohydrate loading were conducted at our department. Analysis of 306 patients with fractures revealed a $71.6 \%$ compliance rate for preoperative carbohydrate loading, and $93.5 \%$ of patients resumed oral liquids within 2 hours after surgery. Compared with non-complying patients who underwent preoperative carbohydrate loading, the preoperative and total fasting times for liquids were significantly shorter in the complying patients. More than $70 \%$ of patients had no thirst or hunger with the current management protocol.

There are many reasons for non-compliance with abbreviated preoperative fasting. Roughead et al. ${ }^{[21]}$ classified patients' compliance as active or passive based on whether the patients were required to make a direct contribution to achieve the compliance. Both education of doctors and nurses and cooperation by patients are needed for successful perioperative fasting management. Additionally, a long and difficult transition in real clinical practice can be predicted. A compliance rate of $>70 \%$ is usually considered satisfactory ${ }^{[22]}$. In this study, $71.6 \%$ of patients underwent preoperative carbohydrate loading. The result was acceptable, although it could still be improved. Nevertheless, the median preoperative fasting time for liquids was still long because of uncertainty of the starting time of the subsequent operation. Because solid food was still forbidden preoperatively on the day of surgery, the preoperative fasting time for solids was not improved.

The benefits of preoperative carbohydrate loading have been verified by many studies and include alleviating insulin resistance ${ }^{[2,11,23]}$, improving patients' subjective feelings ${ }^{[2,4,23,24]}$, and decreasing stress reaction ${ }^{[23-25]}$. Theoretically, a carbohydrate-enriched beverage provides not only provide liquid but also energy. In our study, the patients' thirst and hunger were evaluated and the preoperative fasting time for liquids was an independent risk factor for perioperative hunger. This finding indicates that further shortening of the preoperative fasting time using a carbohydrate-enriched beverage was still needed because $>25 \%$ of patients still experienced different levels of thirst and/or hunger under the current management protocol.

There is no exact time to resume oral nutrition after elective surgery because of variations among the types of surgery performed and the patients' tolerance ${ }^{[8]}$. Long periods of postoperative fasting are still being reported in the literature, especially for patients undergoing digestive tract operations ${ }^{[26,27]}$. However, this is unnecessary according to a study by de Amorim et al., ${ }^{[28]}$ who found that the median postoperative fasting time was reduced to 5.1 hours. For surgeries not involving the gastrointestinal tract, the postoperative fasting time could be even shorter. Ford et al. ${ }^{[29]}$ studied patients' fasting after cardiothoracic surgery and showed that early oral hydration did not increase the incidence of nausea, vomiting, dysphagia, and aspiration pneumonia compared with usual care (a 6-hour fast). Additionally, the number of patients with a high thirst level was significantly reduced in the early oral hydration group. ${ }^{[29]}$ In our study, according to the recommendation of the Chinese guidelines, the median postoperative 
fasting time for liquids and solids was shortened to 1 and 2.8 hours, respectively. No complications such as aspiration pneumonia were observed in this cohort, verifying the safety of this protocol.

This study had some limitations. First, this was an observational study with no control group; thus we could not evaluate the improvement of patients' subjective comfort under the abbreviated fasting protocol. In addition, no biochemical test including stress biomarkers and insulin resistance were included due to the real clinical feature of the study. At last, the preoperative fasting time for both liquids and solids was still long, so further improvement was needed.

\section{Conclusions}

This study involved the implementation of an abbreviated perioperative fasting protocol based on current guidelines and analyzed patients' compliance. Among 306 patients with fresh fractures, a $71.6 \%$ compliance rate for preoperative carbohydrate loading was achieved. The postoperative fasting time was shortened to 1 hour for liquids and 2.8 hours for solids. More than $70 \%$ of patients had no thirst or hunger perioperatively under the current protocol. However, the preoperative fasting time was still relatively long, and further abbreviating the preoperative fasting time would probably further improve patients' subjective comfort according to our logistic regression analysis.

\section{Abbreviations}

ERAS, enhanced recovery after surgery

BMI, body mass index

\section{Declarations}

Competing interests: None of the author of this paper has a financial or personal relationship with other people or organization that could inappropriately influence or bias the content of the paper.

Ethics approval and consent to participate: This study was approved by the ethics committee of Beijing Jishuitan Hospital (Approval No. 201807-12). All patients provided written informed consent to participate. Availability of data and materials: The data and materials could be acquired by contacting the corresponding author with e-mail.

Consent for publication: Not applicable..

Funding: This study was supported by grants of Beijing Municipal Administration of Hospitals Incubating Program (PX2020017) and Capital's Funds for Health Improvement and Research (2018-1-2072).

Authors' contributions: Sun ZJ and Li T analyzed the data and wrote the manuscript. Sun X, Mi M, Zhou Y and Zhao $X$ participated the formulating of abbreviated fasting management. Peng GL, Zhang CL, Jiang Y and Huo Y collected the data. Li T and Wu XB designed the study. All authors reviewed the manuscript. 
Acknowledgment: We thank Angela Morben, DVM, ELS, from Liwen Bianji (Edanz) (www.liwenbianji.cn/) for editing the English text of a draft of this manuscript.

\section{References}

1. Maltby JR. Fasting from midnight--the history behind the dogma. Best Pract Res Clin Anaesthesiol, 2006;20(3):363-78.

2. Shi Y, Dong B, Dong Q, Zhao Z, Yu Y. Effect of Preoperative Oral Carbohydrate Administration on Patients Undergoing Cesarean Section with Epidural Anesthesia: A Pilot Study. J Perianesth Nurs, 2021;36(1):30-35.

3. Aroni P, Fonseca LF, Ciol MA, Margatho AS, Galvão CM. The use of mentholated popsicle to reduce thirst during preoperative fasting: A randomised controlled trial. J Clin Nurs, 2020;29(5-6):840-51.

4. Li T, Zhou Y, Sun X, Sun ZJ, Jiang YH. A prospective cohort study on reducing perioperative fasting in traumatic patients following Enhanced Recovery After Surgery protocol. Chin J Orthop Trauma, 2018;20(4):312-17.

5. Practice Guidelines for Preoperative Fasting and the Use of Pharmacologic Agents to Reduce the Risk of Pulmonary Aspiration: Application to Healthy Patients Undergoing Elective Procedures: An Updated Report by the American Society of Anesthesiologists Task Force on Preoperative Fasting and the Use of Pharmacologic Agents to Reduce the Risk of Pulmonary Aspiration. Anesthesiology, 2017;126(3):376-393.

6. Dobson G, Chong M, Chow L, Flexman A, Kurrek M. Guidelines to the Practice of Anesthesia - Revised Edition 2017. Can J Anaesth, 2017;64(1):65-91.

7. Lambert E, Carey S. Practice Guideline Recommendations on Perioperative Fasting: A Systematic Review. JPEN J Parenter Enteral Nutr, 2016;40(8):1158-65.

8. Weimann A, Braga M, Carli F, Higashiguchi T, Hübner M, et al. ESPEN guideline: Clinical nutrition in surgery. Clin Nutr, 2017;36(3):623-50.

9. Amer MA, Smith MD, Herbison GP, Plank LD, McCall JL. Network meta-analysis of the effect of preoperative carbohydrate loading on recovery after elective surgery. Br J Surg, 2017;104(3):187-97.

10. Pogatschnik C, Steiger E. Review of Preoperative Carbohydrate Loading. Nutr Clin Pract, 2015;30(5):660-4.

11. Smith MD, McCall J, Plank L, Herbison GP, Soop M, et al. Preoperative carbohydrate treatment for enhancing recovery after elective surgery. Cochrane Database Syst Rev, 2014;(8):D9161.

12. Smith I, Kranke P, Murat I, Smith A, O'Sullivan G, et al. Perioperative fasting in adults and children: guidelines from the European Society of Anaesthesiology. Eur J Anaesthesiol, 2011;28(8):556-69.

13. Bethune Alliance of Enhanced Recovery After Surgery in Orthopedics, Trauma Orthopedics Professional Committee of Bethune Charitable Foundation, Joint Surgery Professional Committee of Bethune Charitable Foundation, Society of Enhanced Recovery After Surgery of China International 
Exchange and Promotion Association fro medical and Health Care. Guidelines on perioperative fasting management in orthopeadic surgery. Chin J Orthop Trauma, 2019;21(10):829-34.

14. Johnson RR, Eckert PP, Gilmore W, Viswanath A, Finkelman M, et al. Most American Association of Oral and Maxillofacial Surgeons Members Have Not Adopted the American Society of Anesthesiologists-Recommended Nil Per Os Guidelines. J Oral Maxillofac Surg, 2016; 74(10):192631.

15. Karadag M, Pekin IO. Determining health personnel's application trends of new guidelines for preoperative fasting: findings from a survey. J Perianesth Nurs, 2014;29(3):175-184.

16. Lim HJ, Lee H, Ti LK. An audit of preoperative fasting compliance at a major tertiary referral hospital in Singapore. Singapore Med J, 2014;55(1):18-23.

17. Breuer J P, Bosse G, Seifert S, Prochnow L, Martin J, et al. Pre-operative fasting: a nationwide survey of German anaesthesia departments. Acta Anaesthesiol Scand, 2010;54(3):313-20.

18. Vidot H, Teevan K, Carey S, Strasser S, Shackel N. A prospective audit of preprocedural fasting practices on a transplant ward: when fasting becomes starving. J Clin Nurs, 2016;25(5-6):829-35.

19. Gebremedhn EG, Nagaratnam VB. Audit on preoperative fasting of elective surgical patients in an African academic medical center. World J Surg, 2014;38(9):2200-4.

20. Falconer R, Skouras C, Carter T, Greenway L, Paisley AM. Preoperative fasting: current practice and areas for improvement. Updates Surg, 2014;66(1):31-9.

21. Roughead T, Sewell D, Ryerson C J, Fisher JH, Flexman AM. Internet-Based Resources Frequently Provide Inaccurate and Out-of-Date Recommendations on Preoperative Fasting: A Systematic Review. Anesth Analg, 2016;123(6):1463-8.

22. Brustia R, Mariani P, Sommacale D, Slim K, GRACE Group. The impact of enhanced recovery program compliance after elective liver surgery: Results from a multicenter prospective national registry. Surgery, 2021; 170(5):1457-1466.

23. Rizvanović N, Nesek A V, Čaušević S, Dervišević S, Delibegović S. A randomised controlled study of preoperative oral carbohydrate loading versus fasting in patients undergoing colorectal surgery. Int $\mathrm{J}$ Colorectal Dis, 2019;34(9):1551-61.

24. Zhang Y, Min J. Preoperative Carbohydrate Loading in Gynecological Patients Undergoing Combined Spinal and Epidural Anesthesia. J Invest Surg, 2020;3(7):587-95.

25. Carvalho C, Carvalho A, Preza A, Nogueira P, Mendes K, et al. Metabolic and Inflammatory Benefits of Reducing Preoperative Fasting Time in Pediatric Surgery. Rev Col Bras Cir, 2020;47:e20202353.

26. Francisco SC, Batista ST, Pena G. Fasting in Elective Surgical Patients: Comparison among the Time Prescribed, Performed and Recommended on Perioperative Care Protocols. Arq Bras Cir Dig, 2015;28(4):250-4.

27. Cestonaro T, Madalozzo SM, Thieme R D, Cardoso JN, Campos ACL. The reality of the surgical fasting time in the era of the ERAS protocol. Nutr Hosp, 2014,29(2):437-43. 
28. de Amorim AC, Costa MD, Nunes FL, da Silva MGB, Leão CS, et al. Nutritional Status and Perioperative Fasting Time Versus Complications and Hospital Stray of Surgical Patients. Nutr Hosp, 2015;32(2):878-87.

29. Ford C, McCormick D, Parkosewich J, Derycke-Chapman K, Marshallet J, et al. Safety and Effectiveness of Early Oral Hydration in Patients After Cardiothoracic Surgery. Am J Crit Care, 2020;29(4):292-300. 